\title{
A PROCEDURE FOR SUMMING ASYMPTOTIC SERIES
}

\author{
by R. E. SCRATON \\ (Received 19th October 1968)
}

\section{Introduction}

When a function $f(x)$ possesses an asymptotic series

$$
S(x): a_{0}+a_{1} x^{-1}+a_{2} x^{-2}+a_{3} x^{-3}+\ldots,
$$

this series provides a useful means of evaluating $f(x)$ for large values of $x$. The usual procedure is to sum all the terms in $S(x)$ up to, but excluding, the term of smallest magnitude. The degree of accuracy obtained by this method cannot normally be improved by direct summation of $S(x)$, but sometimes better accuracy can be obtained by using one of the familiar devices for accelerating the convergence of series. Simple $\delta^{2}$-extrapolation may be successful, and Rosser (1) and others have used the Euler transformation to some effect. The method given here provides, in suitable cases, a more effective means of evaluating $f(x)$ from the series for a wide range of values of $x$.

It will be assumed that the $a_{r}$ 's are real and that $x$ is real and positive. It will also be assumed that, although $S(x)$ diverges for all finite $x$, the series

$$
s_{m}(z): a_{0}+\frac{a_{1} z}{(1 !)^{m}}+\frac{a_{2} z^{2}}{(2 !)^{m}}+\frac{a_{3} z^{3}}{(3 !)^{m}}+\ldots
$$

where $z$ is a complex variable, converges in a non-vanishing region for sufficiently large $m$. If the series $s_{m}(z)$ converges for $|z|<\rho$, where $\rho>0$ and $m$ is an integer, but the series $s_{m-1}(z)$ diverges for all non-zero $z$, the series $S(x)$ will be said to be of Type $(m)$. Most series which arise in common practice are of Type (1) or Type (2). Series of Type (1) are considered first; the method is then extended to Type (2) series, and the subsequent generalisation is obvious.

\section{The Borel sum}

A fundamental difficulty in handling asymptotic series is that the series $S(x)$ does not define $f(x)$ uniquely; before proceeding to sum $S(x)$ it is necessary to decide which sum is required. In almost all practical situations in which asymptotic series arise, the required sum is the Borel sum, as defined below.

For a Type (1) series, the series $s_{1}(z)$ given by (1) defines an analytic function $\phi(z)$ for $|z|<\rho$. If $\phi(z)$ has an analytic continuation along the positive real axis, the Borel sum $B(x)$ of $S(x)$ is defined by

$$
B(x)=\int_{0}^{\infty} e^{-t} \phi(t / x) d t
$$


provided, of course, that the integral exists. It will be assumed henceforth that the Borel sum is the sum required, i.e. that $B(x)$ is identical with $f(x)$. Now for any real $c>-1$, it can be seen that

$$
\begin{aligned}
B(x) & =\frac{1}{\Gamma(c+1)} \int_{0}^{\infty} e^{-t} \phi(t / x) d t \int_{0}^{\infty} e^{-u} u^{c} d u \\
& =\frac{1}{\Gamma(c+1)} \int_{0}^{\infty} e^{-T} T^{c+1}\left\{\int_{0}^{1} \phi(T v / x)(1-v)^{c} d v\right\} d T,
\end{aligned}
$$

where $T=t+u$ and $v=t / T$. On integrating by parts and replacing $T$ by $t$, this gives

$$
B(x)=\frac{1}{\Gamma(c+1)} \int_{0}^{\infty} e^{-t} t^{c} \phi(t / x ; c) d t,
$$

where

$$
\phi(z ; c)=\int_{0}^{1}\left\{z v \phi^{\prime}(z v)+(c+1) \phi(z v)\right\}(1-v)^{c} d v .
$$

For $|z|<\rho$,

$$
\begin{aligned}
\phi(z ; c) & =\int_{0}^{1} \sum_{r=0}^{\infty} \frac{a_{r}}{r !}(r+c+1) z^{r} v^{r}(1-v)^{c} d v \\
& =\sum_{r=0}^{\infty} a_{r} \frac{\Gamma(c+1)}{\Gamma(c+r+1)} z^{r} \\
& =a_{0}+\frac{a_{1} z}{(c+1)}+\frac{a_{2} z^{2}}{(c+1)(c+2)}+\frac{a_{3} z^{3}}{(c+1)(c+2)(c+3)}+\ldots
\end{aligned}
$$

the interchange of order of summation and integration being justified by virtue of the uniform convergence of the series for $0 \leqq v \leqq 1$.

It will be noted, of course, that if the function $\phi(z ; c)$ is replaced by the series (5), the integral on the right-hand side of (3) may be integrated formally to give the original asymptotic series. The expression (3) may be regarded as a more general form of the Borel sum (2), to which it reduces when $c=0$.

\section{Use of the $\lambda$-transformation}

The problem of summing $S(x)$ is now reduced to that of evaluating the integral on the right-hand side of (3), with an appropriate value of $c$. As the series (5) defines $\phi(z ; c)$ only for $|z|<\rho$, it is first necessary to find a representation of $\phi(z ; c)$ on the positive real axis; in the treatment which follows, this is obtained by using the $\lambda$-transformation, described elsewhere by the present author (2).

The $\lambda$-transform of the series (5) is the series

$$
\frac{1}{1-\lambda z}\left\{b_{0}+\frac{\lambda z}{1-\lambda z} \Delta b_{0}+\left(\frac{\lambda z}{1-\lambda z}\right)^{2} \Delta^{2} b_{0}+\ldots\right\} \text {, }
$$


where $b_{0}=a_{0}$,

$$
b_{r}=\frac{a_{r}}{(c+1)(c+2) \ldots(c+r) \lambda^{r}}, \quad r \geqq 1
$$

and $\Delta$ is the usual forward difference operator. Wherever this series converges its sum is equal to $\phi(z ; c)$, and for the present purpose it is necessary to find a value of $\lambda$ so that the series converges for all positive real $z$. Reference to (2) will show that this is possible if all the singularities of $\phi(z ; c)$ lie in the region $\operatorname{Re} z \leqq k$, where $k<0$. It will be assumed that this is the case, as in fact it is for many asymptotic series which arise in practice. (One qualification to this last remark is necessary: for a number of familiar series which contain even or odd terms only, some singularities lie on the axis $\operatorname{Re} z=0$. In such cases it is merely necessary to replace $x$ by $x^{\frac{1}{2}}$, so that these singularities are transferred to the negative real axis.) The appropriate value of $\lambda$ will always be real and negative.

Use of the $\lambda$-transformation gives

$$
B(x)=\frac{1}{\Gamma(\dot{c}+1)} \int_{0}^{\infty} e^{-t} t^{c} \sum_{r=0}^{\infty} \frac{(\lambda t / x)^{r}}{(1-\lambda t / x)^{r+1}} \Delta^{r} b_{0} d t .
$$

Assuming, for the moment, that the order of integration and summation can be interchanged,

where

$$
B(x)=\sum_{r=0}^{\infty} K_{r}(-x / \lambda, c)(-1)^{r} \Delta^{r} b_{0},
$$

$$
K_{r}(u, c)=\frac{u}{\Gamma(c+1)} \int_{0}^{\infty} e^{-t} \frac{t^{c+r}}{(u+t)^{r+1}} d t
$$

and $u=-x / \lambda$. The series (8) will be called the $(\lambda, c)$-transform of $S(x)$. It is shown below that $K_{r}(u, c)$ is easily evaluated numerically (in fact it is related to the confluent hypergeometric function, though this is of no concern here) so that the transformation provides a convenient method of summing $S(x)$.

The question of interchanging the order of integration and summation in (7) requires further comment. It is possible that in some circumstances the series may converge, but not converge uniformly, so that the interchange is not justified; indeed in such circumstances it is possible that the series (8) may not converge. It can be shown, however, (though the analysis is too cumbersome to reproduce here) that the interchange is justified if constants $\alpha, \beta$ can be found so that

$$
\left|\Delta^{r} b_{0}\right|<\alpha r^{\beta}
$$

for all $r$. It could possibly be justified under less stringent conditions, but in practice $\Delta^{r} b_{0}$ is usually of approximately constant magnitude, so that the condition (9) will suffice.

The particular case of the $(\lambda, c)$-transformation in which $\lambda=1$ and $c=0$ has been discussed elsewhere, initially by $\operatorname{Ser}(3,4,5)$ and subsequently by van Wijngaarden (6). Both authors make use of tabulated values of $K_{r}(u, 0)$, a suitable table being given in (5). 
4. Calculation of $K_{r}(u, c)$

Let

so that

$$
U_{r}(u, c)=\frac{1}{\Gamma(c+1)} \int_{0}^{\infty} e^{-t} \frac{t^{c+r}}{(u+t)^{r}} d t
$$

It is easily seen that

$$
K_{r}(u, c)=U_{r}(u, c)-U_{r+1}(u, c) .
$$

$$
\begin{gathered}
U_{0}(u, c)=1, \\
U_{r}(u, c) \rightarrow 0 \text { as } r \rightarrow \infty
\end{gathered}
$$

and

$$
(c+r) U_{r-1}(u, c)-(2 r+c+u) U_{r}(u, c)+r U_{r+1}(u, c)=0, \quad r \geqq 1 .
$$

An obvious procedure for calculating $U_{r}(u, c)$ is to evaluate $U_{1}(u, c)$ by quadrature and then use (10) to obtain $U_{2}(u, c), U_{3}(u, c), \ldots$ This is not, however, a satisfactory numerical process. The difference equation (10) has two independent solutions which, for large $r$, are approximately proportional to

$$
r^{t+\frac{1}{2} c} \exp \left[ \pm 2(u r)^{\frac{1}{t}}\right]
$$

the required solution having the negative sign in the exponential. If tabulation is commenced from $U_{0}(u, c), U_{1}(u, c)$, numerical perturbations caused by rounding errors, etc., introduce a multiple of the alternative solution, and since the latter diverges rapidly it ultimately swamps the required solution. If, however, tabulation is carried out in the reverse direction, no such trouble arises since the unwanted solution then dies out. One therefore tabulates the sequence $\left\{P_{r}\right\}$ defined by

$$
\begin{aligned}
P_{n+1} & =1, \\
P_{n} & =\left(\frac{n}{n+1}\right)^{t+\frac{1}{2} c} \exp \left[\left(\frac{u}{n+\frac{1}{2}}\right)^{\frac{1}{2}}\right], \\
P_{r-1} & =\frac{1}{c+r}\left[(2 r+c+u) P_{r}-r P_{r+1}\right], \quad r=n, n-1, \ldots, 2,1,
\end{aligned}
$$

in which $P_{n}$ is chosen so as to be approximately equal to $U_{n}(u, c) / U_{n+1}(u, c)$ when $n$ is large.

Then, for sufficiently large $n, U_{r}(u, c) \bumpeq P_{r} / P_{0}$, and

$$
K_{r}(u, c) \bumpeq \frac{P_{r}-P_{r+1}}{P_{0}}
$$

The approximation is quite good even for small values of $n$, as is shown in the example below.

\section{Numerical example (i)}

Consider the series

$$
S(x): 1-\frac{1}{x}+\frac{1.3}{x^{2}}-\frac{1.3 .5}{x^{3}}+\ldots
$$


which represents the function

$$
f(x)=\left(\frac{\pi x}{2}\right)^{\frac{1}{2}} e^{\frac{1}{x} x} \operatorname{erfc}\left(\sqrt{\frac{x}{2}}\right) .
$$

If $\lambda=-2$ and $c=-\frac{1}{2}$, then $b_{r}=1$ for all $r$, and consequently the $\left(-2,-\frac{1}{2}\right)$ transform of $S(x)$ terminates after only one term; its sum is simply $K_{0}\left(\frac{1}{2} x,-\frac{1}{2}\right)$. (In a simple example such as this, it is easy to show that $f(x)=K_{0}\left(\frac{1}{2} x,-\frac{1}{2}\right)$ without recourse to asymptotic series.) The values obtained for $f(1)$ and $f(5)$ using the procedure described in the previous section with $n=1,2, \ldots, 10$ are shown in Table 1; the correct values are shown at the foot of each column. The power of the method will be appreciated if one compares the values obtained for $f(x)$ with those which could be obtained by direct summation of $S(x)$.

TABLe 1

\begin{tabular}{|r|l|l|}
\hline$n$ & \multicolumn{1}{|c|}{$x=1$} & \multicolumn{1}{|c|}{$x=5$} \\
\hline 1 & 0.652 & 0.86577 \\
2 & 0.6549 & $0.86541,2$ \\
3 & 0.65541 & $0.86539,456$ \\
4 & 0.655575 & $0.86539,2725$ \\
5 & 0.655633 & $0.86539,2629$ \\
6 & 0.655657 & $0.86539,25951$ \\
7 & 0.655668 & $0.86539,25885$ \\
8 & 0.655673 & $0.86539,25870$ \\
9 & 0.655676 & $0.86539,25866$ \\
10 & 0.655678 & $0.86539,25866$ \\
\hline$f(x)$ & 0.655680 & $0.86539,25866$ \\
\hline
\end{tabular}

It is evident that $\lambda$ and $c$ can be chosen so as to make the transformed series terminate whenever $a_{r}$ is of the form

$$
(-1)^{r} a(a+h)(a+2 h) \ldots(a+[r-1] h) k^{r} p(r),
$$

where $a, h, k$ are positive and $p(r)$ is any polynomial in $r$. It might be argued that in such cases one is not really summing the asymptotic series but merely rewriting $f(x)$ as the sum of a finite number of terms which can be evaluated numerically. Certainly the real value of the present method arises when $f(x)$ cannot be put into a form which is readily calculable, but a numerical example in which the transformed series does not terminate will be delayed until Type (2) series have been considered.

\section{Extension to Type (2) series}

If $S(x)$ is of Type (2), the series $s_{2}(z)$ defines an analytic function $\phi(z)$, and the Borel sum of $S(x)$ may be defined as

$$
B(x)=\int_{0}^{\infty} \int_{0}^{\infty} e^{-t} e^{-T} \phi(t T / x) d t d T .
$$

E.M.S. -U 
As before, it can be shown that if $c>-1, d>-1$

$$
B(x)=\frac{1}{\Gamma(c+1) \Gamma(d+1)} \int_{0}^{\infty} \int_{0}^{\infty} e^{-t} e^{-T} t^{c} T^{d} \phi(t T / x ; c, d) d t d T,
$$

where $\phi(z ; c, d)$ is the analytic function defined by the series

$$
\begin{aligned}
a_{0}+\frac{a_{1} z}{(c+1)(d+1)}+\frac{a_{2} z^{2}}{(c+1)(c+2)(d+1)(d+2)} & \\
& +\frac{a_{3} z^{3}}{(c+1)(c+2)(c+3)(d+1)(d+2)(d+3)}+\ldots
\end{aligned}
$$

On applying the $\lambda$-transformation, this gives

where $b_{0}=a_{0}$,

$$
B(x)=\sum_{r=0}^{\infty} K_{r}(-x / \lambda, c, d)(-1)^{r} \Delta^{r} b_{0},
$$

$$
b_{r}=\frac{a_{r}}{(c+1)(c+2) \ldots(c+r)(d+1)(d+2) \ldots(d+r) \lambda^{r}}, \quad r \geqq 1
$$

and

with $u=-x / \lambda$.

$$
K_{r}(u, c, d)=\frac{u}{\Gamma(c+1) \Gamma(d+1)} \int_{0}^{\infty} \int_{0}^{\infty} e^{-t} e^{-r} \frac{t^{c+r} T^{d+r}}{(u+t T)^{r+1}} d t d T,
$$

If

then

$$
U_{r}(u, c, d)=\frac{1}{\Gamma(c+1) \Gamma(d+1)} \int_{0}^{\infty} \int_{0}^{\infty} e^{-t} e^{-T} \frac{t^{c+r} T^{d+r}}{(u+t T)^{r}} d t d T,
$$

and it can be shown that

$$
K_{r}(u, c, d)=U_{r}(u, c, d)-U_{r+1}(u, c, d)
$$

$$
\begin{aligned}
(c+r)(d+r) & U_{r-1}(u, c, d)-\left[3 r^{2}+(2 c+2 d+1) r+c d+u\right] U_{r}(u, c, d) \\
+ & {\left[3 r^{2}+(c+d+2) r\right] U_{r+1}(u, c, d)-r(r+1) U_{r+2}(u, c, d)=0, \quad r \geqq 1 . }
\end{aligned}
$$

Further, for large $r, U_{r}(u, c, d)$ is approximately proportional to

If, therefore, the sequence $\left\{P_{r}\right\}$ is defined by

$$
r^{\frac{1}{3}(c+d+1)} \exp \left[-3(u r)^{\frac{1}{3}}\right] \text {. }
$$

$$
\begin{aligned}
P_{n+1} & =1 \\
P_{n} & =\left(\frac{n}{n+1}\right)^{\frac{1}{3}(c+d+1)} \exp \left[\frac{u^{\frac{1}{3}}}{\left(n+\frac{1}{2}\right)^{\frac{2}{3}}}\right] \\
P_{n-1}= & \left(\frac{n-1}{n+1}\right)^{\frac{1}{3(c+d+1)} \exp \left[\frac{2 u^{\frac{1}{3}}}{n^{\frac{2}{3}}}\right]} \\
P_{r-1}= & \frac{1}{(c+r)(d+r)}\left\{\left[3 r^{2}+(2 c+2 d+1) r+c d+u\right] P_{r}\right. \\
& \left.\quad-\left[3 r^{2}+(c+d+2) r\right] P_{r+1}+r(r+1) P_{r+2}\right\}, \quad r=n-1, n-2, \ldots, 2,1,
\end{aligned}
$$


then, for sufficiently large $n$,

$$
K_{r}(u, c, d) \bumpeq \frac{P_{r}-P_{r+1}}{P_{0}} .
$$

The series (11) will be called the $(\lambda, c, d)$-transform of $S(x)$, and it is clear that this transformed series provides a convenient method of evaluating $f(x)$.

\section{Choice of parameters}

In some cases-as in the numerical example already considered-it is possible to choose values of the parameters $\lambda, c, d$ so that the transformed series terminates. When this is not possible, it is desirable to have some means of choosing the parameters so that the transformed series converges rapidly. Unfortunately it is not possible to give very precise criteria for this choice, but the ideas outlined below may serve as a general guide. The effectiveness of the method is not seriously impaired if values differing appreciably from the best values are used, so that the choice is not crucial.

The method of choosing $\lambda$ so that the series (6) has minimum " convergence ratio" is described fully in (2). If $\phi(z ; c)$ or $\phi(z ; c, d)$ is regular at infinity, the convergence ratio of the series (8) or (11) is the same as the limit of the convergence ratio of (6) as $z \rightarrow \infty$. The value of $\lambda$ should therefore be chosen so as to give optimum convergence of (6) at infinity.

More usually, however, $\phi(z ; c)$ or $\phi(z ; c, d)$ has a singularity at infinity, and further singularities at, say, the set of points

$$
\left\{-\alpha_{s} \pm i \beta_{s}, \alpha_{s}>0, \beta_{s} \geqq 0, s=1,2,3, \ldots\right\} \text {. }
$$

In these circumstances the convergence ratio of (8) or (11) is equal to unity for any value of $\lambda$. The series (6) is used, however, only with positive real values of $z$, and it can be shown that the value

$$
\lambda=-\frac{1}{2 \min _{s}\left(\alpha_{s}\right)}
$$

gives optimum convergence of (6) for sufficiently large positive real $z$ (and indeed for all positive real $z$ if $\alpha_{s} \geqq \beta_{s}$ for all $s$ ). This value of $\lambda$, which is independent of the choice of $c$ and $d$, is therefore recommended, though it is not easy to see how it could be rigorously justified.

The choice of $c$ and $d$ is even more a matter of conjecture. It appears empirically that best results are obtained if these parameters are chosen so that the ratios $\left\{b_{r+1} / b_{r}\right\}$ are approximately constant.

\section{Numerical example (ii)}

Consider the series

$$
S(x): 1-\frac{9}{128} x^{-1}+\frac{3675}{32768} x^{-2}-\frac{2401245}{4194304} x^{-3}+\ldots
$$

in which

$$
a_{r}=\frac{(-1)^{r}[1 \cdot 3 \cdot 5 \ldots(4 r-1)]^{2}}{2^{6 r}(2 r) !} \text {. }
$$


This series represents the function

$$
f(x)=\left(\frac{\pi}{2}\right)^{\frac{1}{2}} x^{\frac{1}{2}}\left[J_{0}\left(x^{\frac{1}{2}}\right) \cos \left(x^{\frac{1}{2}}-\frac{1}{4} \pi\right)+Y_{0}\left(x^{\frac{1}{2}}\right) \sin \left(x^{\frac{1}{2}}-\frac{1}{4} \pi\right)\right] .
$$

The discussion of the preceding section indicates that $\lambda$ should be taken as $-\frac{1}{2}$, and a little trial and error shows that the ratios $b_{r+1} / b_{r}$ can be made very nearly constant by taking $c=d=-0.7382$. The evaluation of $f(1)$ is set out in Table 2; $\Sigma_{r}$ is the sum of the first $(r+1)$ terms of the transformed series. The

TABLE 2

\begin{tabular}{|c|c|c|c|c|c|}
\hline$r$ & $b_{r}$ & $\Delta^{\prime} b_{0}$ & $K_{r}$ & $\Sigma_{r}$ & $\delta^{2}$-extrapolate \\
\hline 0 & 1.000000 & 1.000000 & 0.976667 & 0.976667 & \\
1 & 2.051744 & 1.0517444 & 0.017729 & 0.958021 & \\
2 & 4.110996 & 1.007507 & 0.003369 & 0.961415 & 0.960892 \\
3 & 8.204230 & 1.026475 & 0.001120 & 0.960265 & 0.960556 \\
4 & 16.362678 & 1.004758 & 0.000483 & 0.960751 & 0.960606 \\
5 & 32.634789 & 1.012459 & 0.000243 & 0.960505 & 0.960587 \\
6 & 65.100225 & 1.001537 & 0.000135 & 0.960640 & 0.960592 \\
7 & 129.888376 & 1.002982 & 0.000081 & 0.960559 & 0.960589 \\
8 & 259.202916 & 0.998541 & 0.000051 & 0.960610 & 0.960590 \\
9 & 517.348378 & 0.995910 & 0.000033 & 0.960576 & 0.960590 \\
10 & 1032.740912 & 0.995836 & 0.000023 & 0.960599 & 0.960590 \\
\hline
\end{tabular}

convergence can be improved slightly by using $\delta^{2}$-extrapolation, the results being shown in the final column; the last three entries in this column give $f(1)$ correct to six decimal places. Perhaps more remarkable is that the first three terms in $S(x)$ suffice to give $f(1)$ with an error of only 0.0003 .

\section{REFERENCES}

(1) J. B. Rosser, Transformations to speed the convergence of series. J. Res. Nat. Bur. Stand. 46 (1951), 56.

(2) R. E. Scraton, A note on the summation of divergent power series, Proc. Cambridge Philos. Soc. 66 (1969), 109.

(3) J. SER, Sur la valeur numérique des intégrales employées dans la sommation exponentielle, Bull. Sci. Math. (2) 60 (1936), 199.

(4) J. SER, Quelques applications des polynomes de Laguerre, Bull. Sci. Math. (2) 61 (1937), 74.

(5) J. SER, Formules et tables pour le calcul numérique de certaines séries divergentes. Bull. Math. Sci., (2) 62 (1938), 171.

(6) A. van Wijnganden, A transformation of formal series, Proc. Kon. Ned. Ak. v. Wet., Ser. A, 56 (1953), 522.

\section{UNIVERSITY OF BRADFORD}

BRADFORD 7

YORKS 